\title{
Is it gender, methodology, or something else?
}

\author{
Colleen Gorman Koch, MD, MS ${ }^{\mathrm{a}}$ \\ Christina Mora Mangano, MD $^{\mathrm{b}}$ \\ Nanette Schwann, MD ${ }^{\mathrm{c}}$ \\ Viola Vaccarino, $\mathrm{MD}, \mathrm{PhD}^{\mathrm{d}}$
}

See related articles on pages 929, 936, 950, and 959.

From The Cleveland Clinic Foundation, Cardiothoracic Anesthesia, ${ }^{a}$ Cleveland, Ohio; Stanford University School of Medicine, Department of Anesthesiology, ${ }^{\mathrm{b}}$ Stanford, Calif; Hahnemann University Hospital, Department of Anesthesiology, ${ }^{\mathrm{c}}$ Philadelphia, $\mathrm{Pa}$; and Emory University School of Medicine, Division of Cardiology, ${ }^{\mathrm{d}}$ Atlanta, Ga.

Received for publication June 24, 2003; revisions requested July 7, 2003; revisions received July 16,2003 ; accepted for publication July 21, 2003.

Address for reprints: Colleen Gorman Koch, MD, MS, The Cleveland Clinic Foundation, Cardiothoracic Anesthesia (G3), 9500 Euclid Ave, Cleveland, OH 44195 (E-mail: kochc@ccf.org).

J Thorac Cardiovasc Surg 2003;126:932-5

Copyright $\odot 2003$ by The American Association for Thoracic Surgery

$0022-5223 / 2003 \$ 30.00+0$

doi:10.1016/S0022-5223(03)01304-7

\section{Are Outcomes Different?}

The thesis that women are different from men is not argued in any more important venue than the surgical theatre. The questions of whether and why women have higher probabilities of poor outcomes after coronary artery bypass grafting (CABG) have been repeatedly asked. A common denominator among published investigations is that the preoperative profile of the female patient is vastly different from that of the male patient.

Some risk factors traditionally associated with increased morbidity and mortality after CABG are more commonly represented in the preoperative profile of the female patient. The extent to which these factors explain gender differences, however, varies among studies. Some investigations report higher adjusted mortality for women, ${ }^{1-4}$ whereas others, with application of adjustment strategies, report similar in-hospital mortality between women and men, ${ }^{5-9}$ and yet others report similar postoperative mortality for women and men despite differences in baseline characteristics. $^{10-13}$

How can multiple studies examining the role of gender in surgical outcomes lead to such varied conclusions despite similar analyses of observational databases? If one structures the question correctly, collects the necessary data, and performs the appropriate analysis, one should expect a consistent answer. On the subject of gender, is the question structured to be answered, is there some unmeasured or unknown biologic variable or process-of-care decision in the perioperative period that is influencing outcomes, or is it the variable application of statistical methodology?

\section{Is It Methodology? \\ Study Design}

Understanding the strengths and limitations of experimental versus observational study designs allows for a better understanding of the application of data analysis methods. The randomized controlled trial (RCT) is considered the gold standard when examining outcomes between groups. Specific design elements of the RCT include uniform selection and management of patients under controlled conditions in tertiary care centers. This reduces bias associated with the selection of patients and reduces the variability that results when examining nonhomogeneous populations by balancing known and unknown confounding variables. ${ }^{14-16}$ While reducing variability, these design elements might limit the ability to generalize the results of an RCT for real-world clinical practice. Homogeneous samples of patients might not be reflective of a patient population, thereby limiting treatment efficacy in routine clinical practice. ${ }^{14,16,17}$

On the question of gender, however, an RCT is impossible: one cannot randomize patients to sex. Thus observational databases form the basis for a majority of published investigations. Concato and colleagues ${ }^{18}$ challenged the hierarchical approach to research study designs that consider the observational study to be methodologically inferior to the RCT. This challenges the criticism that all observational studies are weaker or misleading by demonstrating that well-designed observational studies do not systematically overestimate the magnitude of the effects of treatments compared with an RCT. However, data collected for observa- 
tional studies are uncontrolled observations from clinical practice and are therefore prone to selection biases that would be eliminated by the randomization process. ${ }^{17,19}$

Among other concerns are a lack of standardized definitions, richness versus paucity of details on the clinical severity of the comorbid conditions, incompleteness of data collection methods, and a failure to capture physicians' choices regarding process-of-care decisions that are not random. Furthermore, there are temporal trends that occur with advances in surgical technique and medical management that might differentially affect subgroups under investigation. $^{16,20}$

\section{Adjustment Strategies}

How we analyze the data might be the most important source of variability in what has been concluded about the influence of gender on outcomes after CABG. The investigative process among many of the studies generally begins with an examination of patient characteristics by gender, followed by risk adjustment strategies to control for gender differences in preoperative risk factors to determine whether female gender is independently associated with poorer prognosis. Although this is an accepted approach for analysis, there are a number of issues in the statistical techniques used that might explain, in part, the different results obtained in this literature.

Model-free techniques and multivariable modeling. Model-free or statistical modeling techniques are used to adjust for influential variables among patient groups being compared. Many investigations have used model-free techniques, such as matching and subclassification, in an attempt to control for a single or a few factors in the analysis. Others have applied various modeling techniques that model postoperative morbidities or mortality as a function of gender, adjusting for a number of covariables. ${ }^{21}$ The set of covariables selected in the final model is often different across published studies, and this fact might in part explain the variability of results obtained. Factors that could influence variable selection for the final model include the researcher's approach to missing or incorrect data, differences in data coding, and the presence of correlated data. Additional issues that might lead to differences in the final model include the omission of important predictor variables, violations of model assumptions, improper imputation methods, ${ }^{22,23}$ and differences in the approach for variable selection for inclusion in the final model. ${ }^{22}$ Furthermore, there are investigations that omit variables that are not univariably associated with outcome. This might exclude lurking variables that relate to outcome only when other variables that mask their significance are accounted for in the analysis. ${ }^{24,25}$ The application of traditional matching or stratification techniques is ultimately limited by the number of covariates that can be used for matching or stratification. ${ }^{26}$
For example, if a data set contains 200 patients who need to be matched on age, gender, surgical procedure, and a number of comorbid conditions, there might not be enough patients in the sample to match or to stratify on each of the important variables. In addition, regression techniques are at risk of overfitting or underfitting the model if the data contain too few outcome events relative to the number of covariates used for adjustment in the model. ${ }^{27}$ If dealing with a data set of 500 patients who are followed for 12 months, with only 3 deaths occurring during the follow-up period, baseline variables associated with mortality are determined from only a few observations. The regression coefficients for the baseline variables might not be accurate or might be estimated with low precision because of the paucity of outcome events. Underfitting a model can occur when the power to detect important relationships is low. Consider those same 500 patients who are now followed up for a specific event over a 10 -year period, with the development of the event occurring in only 4 patients. Because the outcome events are sparse, an association of risk between a baseline characteristic and a particular outcome would probably be undetectable. Thus the scarcity of outcome events could lead to ambiguous results. ${ }^{27}$ Finally, few investigations provide a measure of model reliability, such as bootstrap aggregation techniques. ${ }^{28}$

Propensity modeling techniques. Propensity modeling allows us to examine patients undergoing CABG from a different statistical vantage point to gain novel insights into the role of gender on postoperative outcomes. ${ }^{29}$ Both propensity score methodology and the parametric approach can provide inferences about causal effects in observational studies. ${ }^{30}$ However, propensity score methods do not share the same limitations as conventional matching, stratification, or multivariable regression techniques in that propensity modeling is not limited by the number of background covariates that can be used. The propensity score provides a summary of the entire spectrum of covariates with one function of the covariates for the individual patient. ${ }^{31,32}$ It is calculated by predicting group membership from the entire spectrum of background characteristics with the use of logistic regression or discriminant analysis. Outcome variables have no role in the prediction of group membership when calculating the propensity score. ${ }^{32}$ The score is calculated by summating the product of the coefficient for each variable by the specific value for each variable. The intercept is then added to this value, resulting in the propensity score for that patient. The propensity score is then the single summarized confounding covariate that can be used for matching, subclassification, or multivariable adjustment. ${ }^{21}$ Patients then matched on propensity scores have a balance in the distribution of covariates, allowing for equivalent comparisons between the groups under investigation. ${ }^{30} \mathrm{~A}$ recent observational investigation of isolated patients un- 
dergoing $\mathrm{CABG}$ applied propensity modeling techniques to examine the role of gender on postoperative morbidity and mortality. Women and men matched on propensity scores had a similar distribution of preoperative comorbid conditions. Most importantly, there was no statistically significant difference in the occurrence of postoperative mortality among the propensity matched pairs of women and men. ${ }^{29}$ Of note, only $26 \%$ of patients were able to be matched on propensity scores. The ability to match only $26 \%$ is reflective of the vastly dissimilar preoperative profiles of women and men undergoing CABG. This investigation demonstrates that, without taking into consideration the dissimilar preoperative profiles of women and men, the mortality results of the same data set can yield different results. In this study, when a standard multivariable logistic regression model for postoperative mortality is applied to the entire data set, irrespective of matching, female gender was significantly predictive of postoperative mortality. A comparison of the distribution of propensity scores among the women and men revealed little overlap in scores. Nothing in standard regression diagnostics will display the lack of overlap among groups under investigation with regard to the distribution of covariates. When the overlap is limited, it is difficult for the data set to support causal conclusions. ${ }^{31}$ Propensity methods, similar to all nonrandomized comparisons, are limited by the potential for omitted confounding variables that might bias the outcome results. ${ }^{30}$

\section{Is it Process-of-Care Decisions?}

Variations in process-of-care decisions in the perioperative period might be contributing to the gender differences in observed outcomes among the published investigations because such variations can influence patient outcomes. Many operative variables, such as cardioplegia techniques, completeness of myocardial revascularization, and decisions to transfuse blood products and administer inotropic agents, are often unmeasured or uncontrolled variables.

There is substantial variability in the perioperative process of care across different institutions and surgical teams. For example, the decision to transfuse a patient perioperatively in many cases is based on anecdotal practices of the particular cardiac team and institution. In a recent observational investigation on the role of gender in the outcomes of CABG, $76 \%$ of women were transfused red blood cells in the operative and postoperative periods. ${ }^{29}$ Furthermore, among the propensity matched pairs of women and men, women were transfused red blood cells more frequently than men despite similar preoperative blood volumes, hematocrit levels, body surface areas, and rates of re-exploration for bleeding. ${ }^{29}$ The process-of-care decision to transfuse red blood cells is an unaddressed variable in many of the investigations of gender on outcomes after CABG.
Another process-of-care issue that might influence patient outcomes and therefore gender differences in outcomes is the decision to administer inotropic agents in the perioperative period. It has been reported that women require more inotropic agents ${ }^{13,29}$ and have a greater occurrence of cardiac morbidity postoperatively, despite equal or better preserved left ventricular function and equal or less extensive coronary artery disease preoperatively. 3,11,29

Is there an unmeasured process-of-care decision in the operating room the result of which is the perception of myocardial injury in the postoperative period? Could it be related to the more frequent occurrence of blood transfusions in women or to different approaches to myocardial protection or completeness of myocardial revascularization? Process-of-care decisions are being made that we do not fully understand and might be contributing to the problem and the apparent differences in outcomes. In these instances, although we are clearly managing women differently from men, there is not a sufficient scientific basis to justify these different treatment approaches.

\section{Is it Biologic Mystique?}

Biologic variability among women and men with regard to the pharmacokinetic and pharmacodynamic profiles for a number of drugs has been demonstrated. However, there is an insufficient amount of information to determine whether these differences in response influence outcomes. ${ }^{33}$ Is the demonstrated need for prolonged postoperative ventilatory support in women after $\mathrm{CABG}^{29,34}$ the result of gender differences in the pharmacodynamic or pharmacokinetic response to drugs used in the perioperative period? In general, narcotic, benzodiazepine, and muscle relaxant drugs given in the perioperative period are arbitrarily dosed without taking into account body weight, unless as part of a clinical trial. The fact that women receive equivalent doses of medications as men without considering ideal body weight might contribute to the observed gender differences in postoperative ventilatory support after CABG. Certainly the influence of hormones, stages of the menstrual cycle, amount and distribution of adipose tissue, and pharmacodynamic and pharmacokinetic differences between women and men in response to perioperative medications requires further investigation.

\section{Answers?}

Variable application of statistical techniques and unmeasured factors, particularly process-of-care decisions, might indeed have a strong influence on the variability in the reported results that have been attributed to gender differences in outcome. The effect of unaccounted biologic factors among women and men begs for further investigation. Although the superiority of one sex can (and will) be argued, we should make all the possible efforts, scientifi- 
cally and technologically, so that equal operative outcomes can be obtained in women and men.

\section{References}

1. Edwards F, Carey J, Grover F, Bero J, Hartz R. Impact of gender on coronary bypass operative mortality. Ann Thorac Surg. 1998;66:12531 .

2. Vaccarino V, Abramson J, Veledar E, Weintraub W. Sex differences in hospital mortality after coronary artery bypass surgery: evidence for a higher mortality in younger women. Circulation. 2002;105:1176-81.

3. Brandrup-Wognsen G, Berggren H, Hartford M, Hjalmarson A, Karlsson T, Herlitz J. Female sex is associated with increased mortality and morbidity early, but not late, after coronary artery bypass grafting. Eur Heart J. 1996;17:1426-31.

4. Christakis G, Weisel R, Buth K, Fremes S, Rao V, Panagiotopoulos K, et al. Is body size the cause for poor outcomes of coronary artery bypass operations in women? J Thorac Cardiovasc Surg. 1995;110: 1344-58.

5. Woods S, Noble G, Smith J, Hasselfeld K. The influence of gender in patients undergoing coronary artery bypass graft surgery: an eight-year prospective hospitalized cohort study. J Am Coll Surg. 2003;196:42834.

6. O'Connor G, Morton J, Diehl M, Olmstead E, Coffin L, Levy D, et al. Differences between men and women in hospital mortality associated with coronary artery bypass graft surgery. Circulation. 1993;88:210410 .

7. Koch C, Higgins T, Capdeville M, Maryland P, Starr N. The risk of coronary artery surgery in women: a matched comparison using preoperative severity of illness scoring. J Cardiothorac Vasc Anesth. 1996;10:839-43.

8. Khan S, Nessim S, Gray R, Czer L, Chaux A, Matloff J. Increased mortality of women in coronary artery bypass surgery: evidence for a referral bias. Ann Intern Med. 1990;112:561-7.

9. Fisher L, Kennedy J, Davis K, Maynard C, Fritz J, Kaiser G, Myers W. Association of sex, physical size and operative mortality after coronary artery bypass in the coronary artery surgery study (CASS). $J$ Thorac Cardiovasc Surg. 1982;84:334-41.

10. Mickleborough L, Takagi Y, Maruyama H, Sun Z, Mohamed S. Is sex a factor in determining operative risk for aortocoronary bypass graft surgery? Circulation. 1995;92:II80-4.

11. Abramov D, Tamzriz M, Sever J, Christakis G, Bhatnagar G, Heenan $\mathrm{A}$, et al. The influence of gender on the outcome of coronary artery bypass surgery. Ann Thorac Surg. 2000;70:800-6.

12. Jacobs A, Kelsey S, Brooks M, Faxon D, Chaitman B, Bittner V, et al. Better outcome for women compared with men undergoing coronary revascularization. A report from the bypass angioplasty revascularization investigation (BARI). Circulation. 1998;98:1279-85.

13. Aldea GS, Gaudiani JM, Shapira OM, Jacobs A, Weinberg J, Cupples A, et al. Effect of gender on postoperative outcomes and hospital stays after coronary artery bypass grafting. Ann Thorac Surg. 1999;67:1097103.

14. Singer AJ, Thode HC, Hollander JE. Research fundamentals: selection and development of clinical outcome measures. Acad Emerg Med. 2000;7:397-401.
15. Pocock SJ. The justification for randomized controlled trials. In: Pocock SJ, editor. Clinical trials: a practical approach. New York: John Wiley \& Sons; 1983. p. 50-65.

16. Simon G, Wagner E, Vonkorff M. Cost-effectiveness comparisons using "real world" randomized trials: the case of new antidepressant drugs. J Clin Epidemiol. 1995;48:363-73.

17. Johanson J. Outcomes research, practice guidelines, and disease management in clinical gastroenterology. J Clin Gastroenterol. 1998;27: 306-11.

18. Concato J, Shah N, Horwitz R. Randomized, controlled trials, observational studies and the hierarchy of research designs. $N$ Engl J Med. 2000;342:1887-92.

19. Pocock S, Elbourne D. Randomized trials or observational tribulations. N Engl J Med. 2000;342:1907-9.

20. Bourne R. Con: some clinical research is not cost-effective-weaknesses of outcomes studies. Spine. 1995;20:386-7.

21. Blackstone EH. Comparing apples and oranges. J Thorac Cardiovasc Surg. 2002;123:8-15.

22. Naftel D. Do different investigators sometimes produce different multivariable equations from the same data? J Thorac Cardiovasc Surg. 1994;107:1528-9.

23. Harrell F, Lee K, Mark D. Tutorial in biostatistics: multivariable prognostic models: issues in developing models, evaluating assumptions and adequacy, and measuring and reducing errors. Stat Med. 1996;15:361-87.

24. Joiner BL. Lurking variables: some examples. Am Stat. 1981;35:22733.

25. Gum PA, Thamilarasan M, Watanabe J, Blackstone E, Lauer M. Aspirin use and all-cause mortality among patients being evaluated for known or suspected coronary artery disease: a propensity matched analysis. JAMA. 2001;286:1187-94.

26. D'Agostino R. Tutorial in biostatistics: propensity score methods for bias reduction in the comparison of a treatment to a non-randomized control group. Stat Med. 1998;17:2265-81.

27. Concato J, Feinstein A, Holford T. The risk of determining risk with multivariable models. Ann Intern Med. 1993;118:201-10.

28. Breiman L. Bagging predictors. Machine Learning. 1996;24:123-40.

29. Koch CG, Khandwala F, Nussimeier N, Blackstone E. Gender and outcomes after coronary artery bypass grafting: a propensity matched comparison. J Thorac Cardiovasc Surg. 2003. In press.

30. Drake C, Fisher L. Prognostic models and the propensity score. Int $J$ Epidemiol. 1995;24:183-7.

31. Rubin D. Estimating causal effects from large data sets using propensity scores. Ann Intern Med. 1997;127:757-63.

32. Rosenbaum P, Rubin D. The central role of the propensity score in observational studies for causal effects. Biometrika. 1983;70:41-55.

33. Wizemann T, Pardue M, editors. Sex affects health. In: Exploring the biological contributions to human health: does sex matter? Washington, DC: Institute of Medicine, National Academy Press; 2001. p. 117-72.

34. Butterworth J, James R, Prielipp R, Cerese J, Livingston J, Burnett D. Female gender associates with increased duration of intubation and length of stay after coronary artery surgery. Anesthesiology. 2000;92: 414-24. 